\title{
Small RNA sequencing reveals various microRNAs involved in piperine biosynthesis in black pepper (Piper nigrum L.)
}

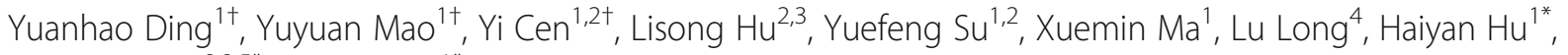
Chaoyun $\mathrm{HaO}^{2,3,5^{*}}$ and Jie Luo ${ }^{1 *}$

\begin{abstract}
Background: Black pepper (Piper nigrum L.), an important and long-cultivated spice crop, is native to South India and grown in the tropics. Piperine is the main pungent and bioactive alkaloid in the berries of black pepper, but the molecular mechanism for piperine biosynthesis has not been determined. MicroRNAs (miRNAs), which are classical endogenous noncoding small RNAs, play important roles in regulating secondary metabolism in many species, but less is known regarding black pepper or piperine biosynthesis.

Results: To dissect the functions of miRNAs in secondary metabolism especially in piperine biosynthesis, 110 known miRNAs, 18 novel miRNAs and 1007 individual targets were identified from different tissues of black pepper by small RNA sequencing. QRT-PCR and 5'-RLM-RACE experiments were conducted to validate the reliability of the sequencing data and predicted targets. We found 3 miRNAs along with their targets including miR166-4CL, miR396PER and miR397-CCR modules that are involved in piperine biosynthesis.

Conclusion: MiRNA regulation of secondary metabolism is a common phenomenon in plants. Our study revealed new miRNAs that regulate piperine biosynthesis, which are special alkaloids in the piper genus, and they might be useful for future piperine genetic improvement of black pepper.
\end{abstract}

Keywords: Black pepper, miRNA, Secondary metabolism, Alkaloids, Piperine biosynthesis

\section{Background}

Black pepper (Piper nigrum L.) is well-known as the king of spices and was known as 'black gold' in ancient times due to its high commercial value and worldwide use in flavouring food. As a traditional medicinal plant, black pepper is widely used in the treatment of pain, chills, rheumatism, muscular aches, and exhaustion and to

\footnotetext{
*Correspondence: yanhai0987@163.com; cyhao_catas@163.com; jie.luo@hainanu.edu.cn

${ }^{+}$Yuanhao Ding, Yuyuan Mao and Yi Cen contributed equally to this work. 'Hainan Key Laboratory for Sustainable Utilization of Tropical Bioresource, School of Tropical Crops, Hainan University, Haikou 570228, China

${ }^{2}$ Spice and Beverage Research Institute, Chinese Academy of Tropical Agricultural Sciences, Wanning 571533, Hainan, China

Full list of author information is available at the end of the article
}

stimulate appetite [1]. As the main economic component of this plant, the spicy berries of black pepper contain extensive alkaloids, such as piperine, pellitorine, piperidine, dehydropipernolanine, piperloein $\mathrm{B}$ and pipernonaline. Piperine is the major pungent and principle bioactive constituent of black pepper, but the molecular mechanism and regulatory network for piperine biosynthesis have not been elucidated.

The biosynthesis of piperine originates from the condensation of piperidine with piperoyl-CoA. An enzyme called 'piperoyl-CoA:piperidine $N$-piperoyltransferase' catalyses the synthesis of piperine in black pepper, but the gene and protein sequences of this enzyme have not been determined. Piperidine synthesis in black pepper is

(C) The Author(s). 2021 Open Access This article is licensed under a Creative Commons Attribution 4.0 International License, which permits use, sharing, adaptation, distribution and reproduction in any medium or format, as long as you give appropriate credit to the original author(s) and the source, provide a link to the Creative Commons licence, and indicate if changes were made. The images or other third party material in this article are included in the article's Creative Commons licence, unless indicated otherwise in a credit line to the material. If material is not included in the article's Creative Commons licence and your intended use is not permitted by statutory regulation or exceeds the permitted use, you will need to obtain permission directly from the copyright holder. To view a copy of this licence, visit http://creativecommons.org/licenses/by/4.0/ The Creative Commons Public Domain Dedication waiver (http://creativecommons.org/publicdomain/zero/1.0/) applies to the data made available in this article, unless otherwise stated in a credit line to the data. 
from the amino acid lysine, which is first decarboxylated to cadaverine by the enzyme lysine decarboxylase (LDC) in the presence of pyridoxal phosphate (PLP). Cadaverine undergoes oxidative deamination via amine oxidase to a 5-aminopentanal, which is then cyclized to yield the $\Delta^{1}$-piperideine. Subsequently, $\Delta^{1}$-piperideine is reduced to piperidine, but the enzymes catalysing 5aminopentanal to piperidine have not been identified. Piperoyl-CoA is derived from $p$-coumaric acid, which is generated from phenylalanine in the presence of oxygen and NADPH or tyrosine via the corresponding phenylalanine ammonia lyase (PAL). Cinnamoyl-CoA, derived from $p$-coumaric acid, is an important precursor for piperoyl-CoA biosynthesis. The piperoyl moiety is generated by the cyclization of meta-methoxy and para-hydroxyl groups on the cinnamoyl backbone. Then, a keto-ester is generated by the chain elongation of cinnamoyl-CoA with acetyl-CoA or malonylCoA via a Claisen-type reaction, which is then reduced by NADPH and followed by dehydration to afford piperoyl-CoA [2-4].

MiRNAs are a class of endogenous small noncoding RNAs that are known as regulators of gene expression at the posttranscriptional level and function in the biosynthesis of secondary metabolites in plants [5]. In Arabidopsis, miR156-targeted SPL9 negatively regulates anthocyanin accumulation by repressing the expression of flavonoid 3'-hydroxylase $\left(F 3^{\prime} H\right)$, dihydroflavonol reductase $(D F R)$, and other anthocyanin biosynthetic genes through a MYB-bHLH-WD40 transcriptional activation complex [6]. Recently, miR828 and miR858 targeting MYB114 were found to promote anthocyanin biosynthesis in high anthocyanin grape lines [7]. Moreover, numerous miRNAs, such as miR172i, miR5298b, miR396b and miR828a, were found to be involved in phenylpropanoid biosynthesis in Podophyllum hexandrum [8], Taxus [9] and Diospyros kaki Thunb [10]. For terpenoid biosynthesis, miR156-targeted SPL9 can positively regulate the expression of terpene synthase 21 (TPS21) by directly binding its promoter, thereby controlling the synthesis of sesquiterpenoids [11]. As nitrogen-containing low-molecular-weight compounds, alkaloids are derived from amino acids and are highly diverse and heterogeneous in nature. It has been reported that some miRNAs participate in alkaloid biosynthesis, such as benzylisoquinoline in opium poppy and nicotine in tobacco $[12,13]$.

Till the date, only a few miRNA studies have investigated the Piper genus [14-17]. Most of these studies have been focused on aspects, such as miRNA structure, family belonging and conservatism, but there is little known about their functions in regulating developmental processes and in controlling metabolism in black pepper. In this study, high-quality small RNA sequencing was performed to identify the temporal and spatial expression of miRNAs and their regulation in controlling metabolism in black pepper. A total of 24 samples were prepared, including 3 biological replicates of 8 different tissues of root, stem, leaf, flower and 4 stages of fruits. The results showed that nearly two-thirds of known miRNAs are specifically up- or downregulated in fruits. Functional analyses have suggested that some miRNAs and their corresponding targets might be involved in black pepper resistance to biotic stress and piperine biosynthesis. Our findings indicated the potential functions of miRNAs in black pepper in regulating the biosynthesis of various secondary metabolites and provide new insights into miRNA functions in black pepper.

\section{Results}

High-throughput small RNA sequencing of $P$. nigrum

To identify miRNAs potentially involved in secondary metabolism especially in alkaloid metabolism in $P$. nigrum, we constructed 24 small RNA libraries including the roots $(\mathrm{R})$, stems $(\mathrm{S})$, leaves $(\mathrm{L})$, flowers $(\mathrm{FL})$ and 4 stages of fruits (FR1-4, fruits after flowering for 2, 4, 6 and 8 months) from $P$. nigrum cultivar Reyin1 with three biological replicates. Nearly 11.6 million raw reads and 9.9 million clean reads $(\sim 84.8 \%)$ were finally obtained (Fig. 1A). In this study, $80 \%$ of clean tags were mapped to the $P$. nigrum genome, which was then used for known and novel miRNA identification, and small RNA annotation (Fig. 1B). The results showed that reads from intergenic regions, repeats, exons, introns and rRNA together occupied most $(\sim 86 \%)$ of the mapped reads, and $1.14 \%$ of the mapped reads were annotated as miRNAs (Fig. 1C). Small RNA lengths of clean reads ranging from 18 to 35 nt were counted and are shown in Fig. 2A. The highest and second abundances were at 24 and 21 nt lengths in all samples, indicating a normal distribution of small RNA lengths compared to other small RNA sequencing studies $[18,19]$.

\section{Known and novel miRNAs identification in P. nigrum}

To illustrate miRNAs involved in secondary metabolism, known and novel miRNAs were identified using clean reads mapped to the $P$. nigrum genome (methods described in the 'Bioinformatics analysis' section). In total, 128miRNAs, including 110 known and 18 novel miRNAs, were detected from small RNA sequencing data (Table 1, Supplementary Table 1). In each sample, nearly 90 known and 14 novel expressed miRNAs could be detected (Table 1). A Venn diagram was made to show the expression of miRNAs in each sample (miRNAs from 4 samples of fruit were merged together, Fig. 2B). Most miRNAs (91/128) were found to be commonly expressed in all samples, and tissue-specific expressed miRNAs occupied a minor part (Fig. 2B). The expression levels of 


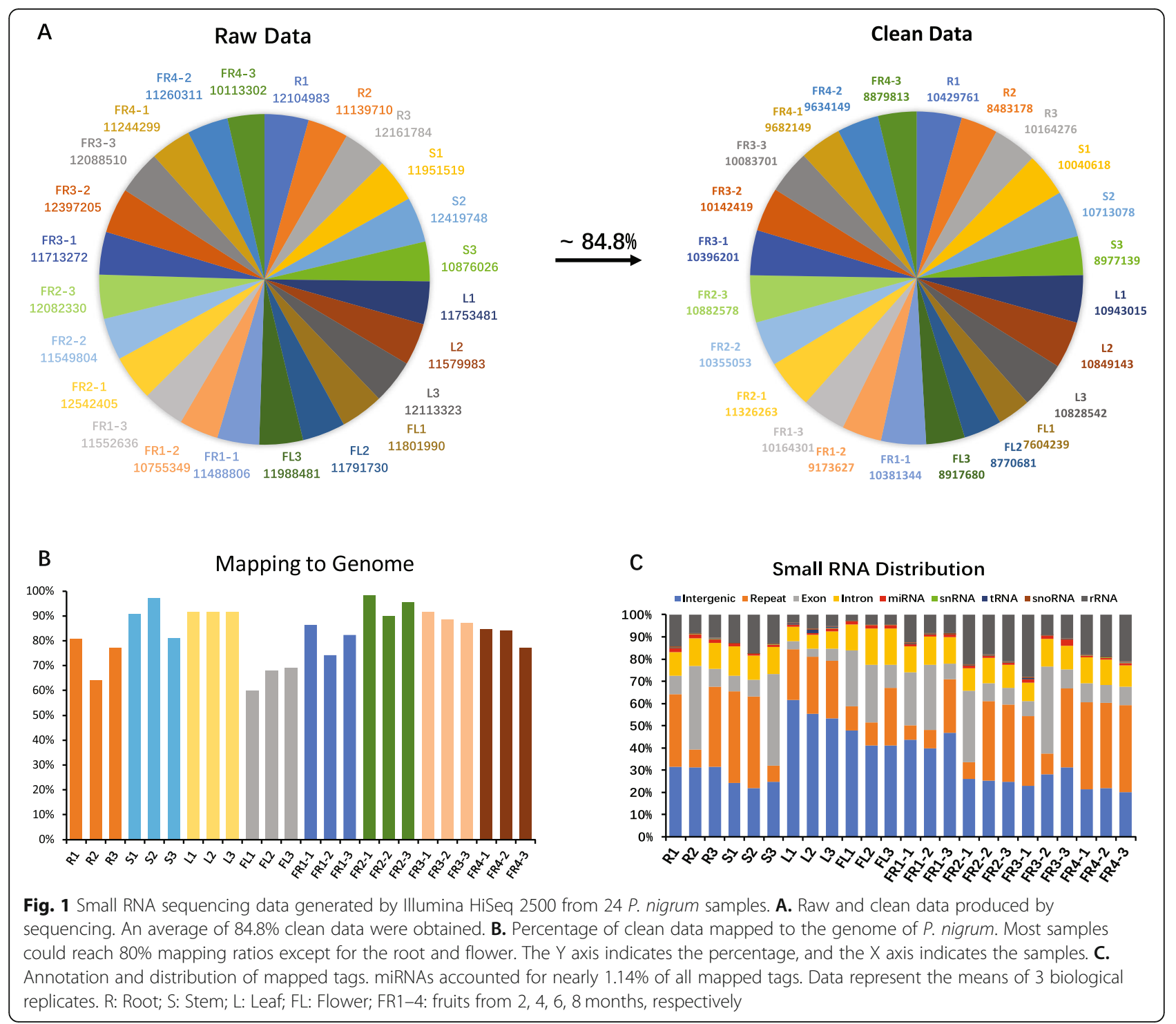

miRNAs were then calculated as transcripts per kilobase million (TPM). The numbers of known and novel miRNAs in each sample were counted according to TPM values ranging from 0 to $>100$ and are shown in Fig. 2C. Among the known miRNAs, miRNAs with low expression $(\mathrm{TPM}<20)$ occupied the most part $(>80$ in 110$)$, and only $\sim 10$ miRNAs were highly expressed (TPM > 100). Among the novel miRNAs, the amount of lowand highly expressed miRNAs was similar in all samples (Fig. 2C). These results reveal that highly expressed miRNAs are considered occupied the minor part in $P$. nigrum, which is also reflected in Supplementary Table 1 showing TPM values.

To further classify the miRNAs, the miRNAs with low expression with $\mathrm{TPM}<5$ in all samples were first removed, leaving 68 known and 16 novel differentially expressed miRNAs (DEMs, Table 1). The fruit of black pepper is rich in notable secondary metabolites, especially alkaloids, compared with other tissues. To understand the miRNA expression patterns between fruits and other tissues (R, S, L, FL), a heatmap of known and novel miRNAs (Fig. 3) was made using Genesis [20] (http://genome.tugraz.at/) using the $\log _{2}$ (fold changes) values (Supplementary Table 2). The results showed that DEMs between fruits and other tissues were clustered together, indicating special expression patterns between samples. All 68 known miRNAs were divided into 3 clusters (Type I-III) including 23 up- (Type I) and 24 down- (Type II) regulated DEMs in fruits, which together occupied nearly $2 / 3$ of the DEMs in the known miRNAs (Fig. 3). The remaining DEMs (Type III, 21) did not show obvious expression trends between tissues. For 16 novel miRNAs, only novel_miR9 was found downregulated in the 4 stages of fruit. Novel_miR10 and 

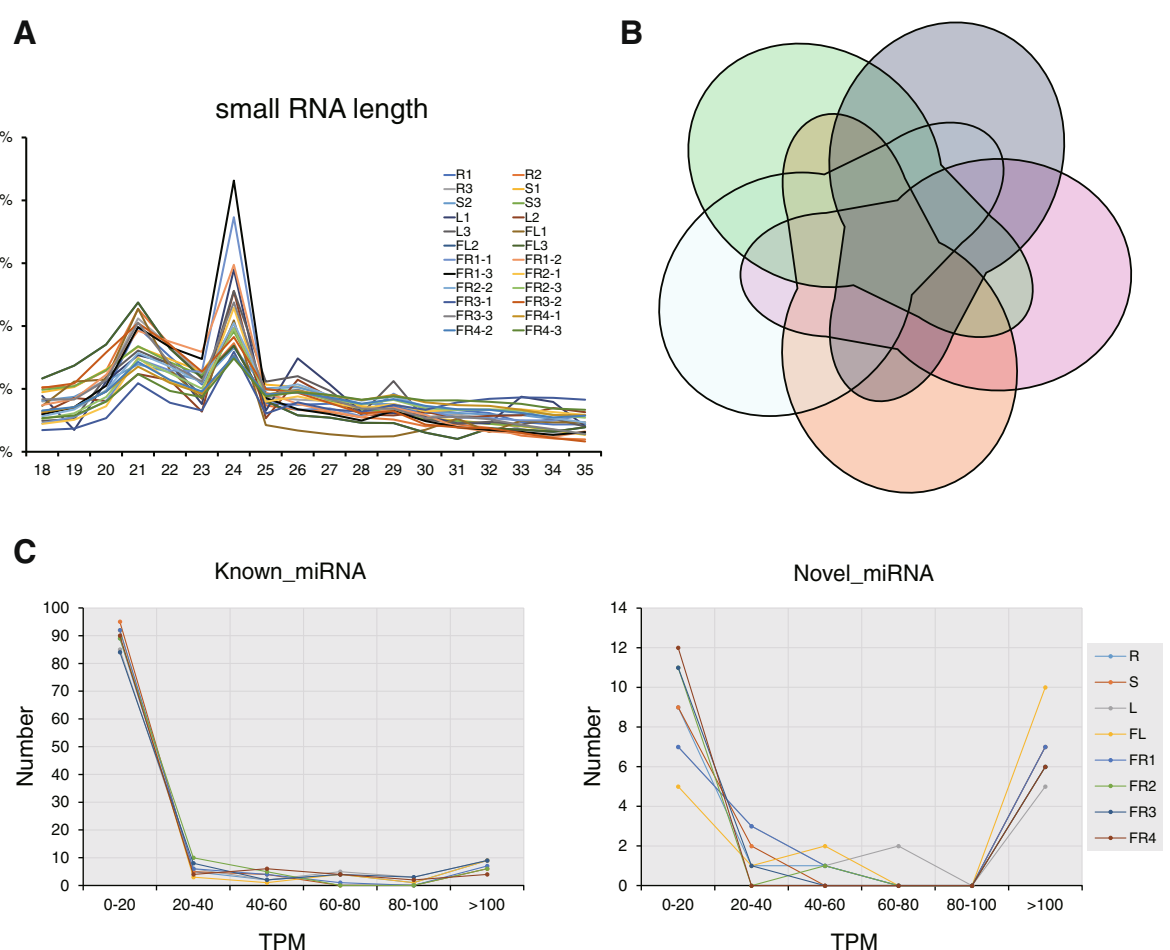

Fig. 2 Small RNA length distribution, specifically expressed miRNA distribution and expression level ranges of known and novel miRNAs in $P$. nigrum. A. Small RNA length in all samples from 18 to $35 \mathrm{nt}$. B. Specific expressed miRNAs distributed in different tissues. miRNAs from 4 stages of fruits (FR1-4) were merged together to FR represented miRNAs expressed in the fruit. C. Number of identified known and novel miRNAs in P. nigrum. X-axis represents the ranges of TPM values, $y$-axis represent the numbers of miRNAs

novel_miR18 were found specially expressed in root and flower, respectively (Fig. 3). Furthermore, 6 novel miRNAs (novel_miR12 - miR17) were found highly expressed in leaves and flowers. The remaining novel miRNAs were downregulated or upregulated in 1 or 2 tissues.

Table 1 Summary of known and novel miRNAs as detected by small RNA sequencing of $P$. nigrum

\begin{tabular}{llllll}
\hline & \multicolumn{2}{l}{ \#number (All) } & & \multicolumn{2}{l}{ \#number (TPM > 5) } \\
\cline { 2 - 3 } & Known miRNA & Novel miRNA & & Known miRNA & Novel miRNA \\
\hline R & 87 & 14 & 34 & 13 \\
$\mathrm{~S}$ & 89 & 13 & 30 & 13 \\
$\mathrm{~L}$ & 89 & 13 & 41 & 13 \\
FL & 90 & 17 & 42 & 15 \\
FR-1 & 98 & 16 & 39 & 14 \\
FR-2 & 92 & 14 & 38 & 14 \\
FR-3 & 92 & 14 & 43 & 14 \\
FR-4 & 92 & 13 & 29 & 13 \\
Total & 110 & 18 & 68 & 16 \\
\hline
\end{tabular}

\section{Expression validation of the miRNA expression profiles via qRT-PCR}

A total of 15 miRNAs (including 10 known and 5 novel miRNAs) with high or low expression levels were selected randomly to perform qRT-PCR to validate the reliability of the small RNA sequencing. $\log _{2}$ (ratios) of qRT-PCR and small RNA-Seq were used to show the correlation between each other. Overall, qRT-PCR measurements were generally in agreement with the small RNA sequencing $\left(R^{2}=0.8706\right.$; Fig. $\left.4 \mathrm{~A}\right)$. The qRT-PCR results of 4 relatively highly expressed miRNAs derived by small RNA sequencing are shown in Fig. 4B. We found that the expression levels of four miRNAs were relatively high according to small RNA sequencing. The transcript of miR156 increased gradually from R to FR; the expression of miR166 was extremely high in FL; miR398 was preferentially expressed in FL and the fruits; and miR396 showed a relatively high expression level in all tissues but decreased gradually from R to FR1. Additionally, the expression level of 4 novel miRNAs were also detected and showed in Fig. S1. We found that novel miR5, novel miR7 and novel miR18 were both highly expression in FL, and novel miR9 highly expressed in R, S and L, which was largely corresponding to the trends of TPM values (Fig. S1). 


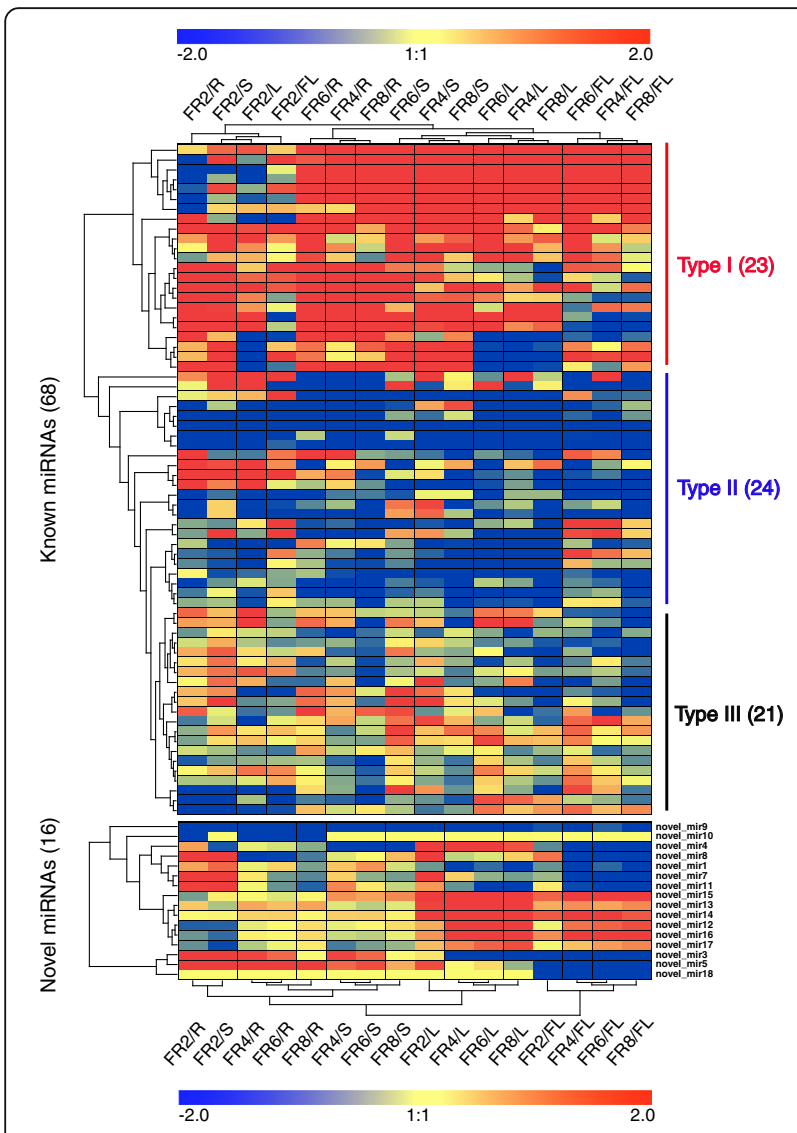

Fig. 3 Heatmaps of known and novel miRNAs. Heatmap showing fold changes of 68 known miRNAs and 16 novel miRNAs between tissues. For known miRNAs, 3 miRNA clusters (type I-III) were classified, including nearly $1 / 3$ upregulated (23/68, type I) and $1 / 3$ downregulated (24/68, type II) DEMs in fruits and 1/3 DEMs (21, type III) without obvious expression trends in tissues. Heatmap showing fold changes of 16 novel miRNAs between tissues. Log $_{2}$ (fold change ratios) of fruits to other tissues were used. Red indicates upregulation, blue means downregulation and yellow indicates no change. The heatmap was made by Genesis

\section{MiRNA target prediction; GO and KEGG pathway analysis} The function of miRNAs depends on the posttranscriptional regulation of their targets; therefore, target prediction and validation are the basis for learning the function of miRNAs. A total of 1007 individual targets (including 2722 cleavage sites) of 125 miRNAs were predicted by TargetFinder and psRobot (Fig. 5A, Supplementary Tables 3,4). In total, $87.79 \%$ of targets were annotated, including GO [21] ( 31.97\%) and KEGG [22] $(\sim 32.37 \%)$ annotations. All these annotated targets were then used for Blast2GO and KEGG pathway enrichment analysis to classify their functions (Supplementary Table 5 and 6). We were interested in the miRNAs regulating the targets that are related to secondary metabolism; thus, correlatively enriched metabolic pathways were considered (Fig. 5B). Our results showed that many metabolic processes were significantly enriched (highlighted by red ticks) such as phenylpropanoid metabolism, lignin metabolism, and cyclic compound biosynthetic processes. Additionally, the transcriptional processes were highly enriched, such as the regulation of gene expression, nucleic acid-templated transcription, and transcription processes (Fig. 5B), which implied that miRNAs and their targets participated in various metabolic processes. Furthermore, targets of miRNAs on molecular functions were clearly enriched in DNA and nucleic acid binding activity, and the most enriched cellular component was the nucleus (Fig. 5B), indicating that enriched targets may function in regulating gene transcription and expression. Taken together, our results suggest that these predicted targets of our miRNAs are likely to regulate metabolic processes and gene expression.

According to KEGG analysis, the most enriched pathways were plant hormone signal transduction, transcription factors and plant-pathogen interactions (Supplementary Fig. 2), which implied that many miRNAs and their targets participated in the response to biotic stress in black pepper. The candidate targets of miRNAs include transcription factors (TFs), such as MYB and ARF, and some important proteins in disease resistance and hormone signal transduction, such as RPS2, PRM1, BRI1, TIR1 and DELLA (Supplementary Table 7). For black pepper living in tropical environmental conditions, enriched plant-pathogen interactions and plant hormone signal transduction pathways are reasonable. Furthermore, all 1007 targets were then used for TF prediction using PlantTFDB [23] (http://planttfdb.cbi.pku.edu.cn/). There were 5 highly abundant TF families, MYB (34), SBP (19), ARF (18), GRF (15) and NAC (11) (Supplementary Table 8). These TFs are mostly involved in the processes of plant growth and abiotic or biotic stress responses, indicating that many miRNAs and their targets might participate in the response to biotic stress resistance in black pepper. In addition, 111 targets occupying nearly $22.6 \%$ of the KEGG annotated targets (326) of 74 miRNAs were found to be involved in various metabolic pathways (Supplementary Table 7). The miRNAs and their corresponding targets related to the following pathways are listed in Table 2: phenylpropanoid biosynthesis; tropane, piperidine and pyridine alkaloid biosynthesis; isoquinoline alkaloid biosynthesis; anthocyanin biosynthesis; and phenylalanine, tyrosine and tryptophan biosynthesis. Genes such as $4 C L$ and $C U A O$ were found to participate in various metabolic processes. In addition, the homologous genes of 4 PERs (Pn24.105, Pn7.365, Pn16.989 and Pn4.2519) involved in catalysing the synthesis of lignin monomers were predicted as the targets of miR396 (Supplementary Fig. 3A), the binding site located at a relatively conserved region of $P E R$ that encodes the amino acid sequences of E/QCPGVVS (Supplementary Fig. 3B). Furthermore, 2 miRNAs 


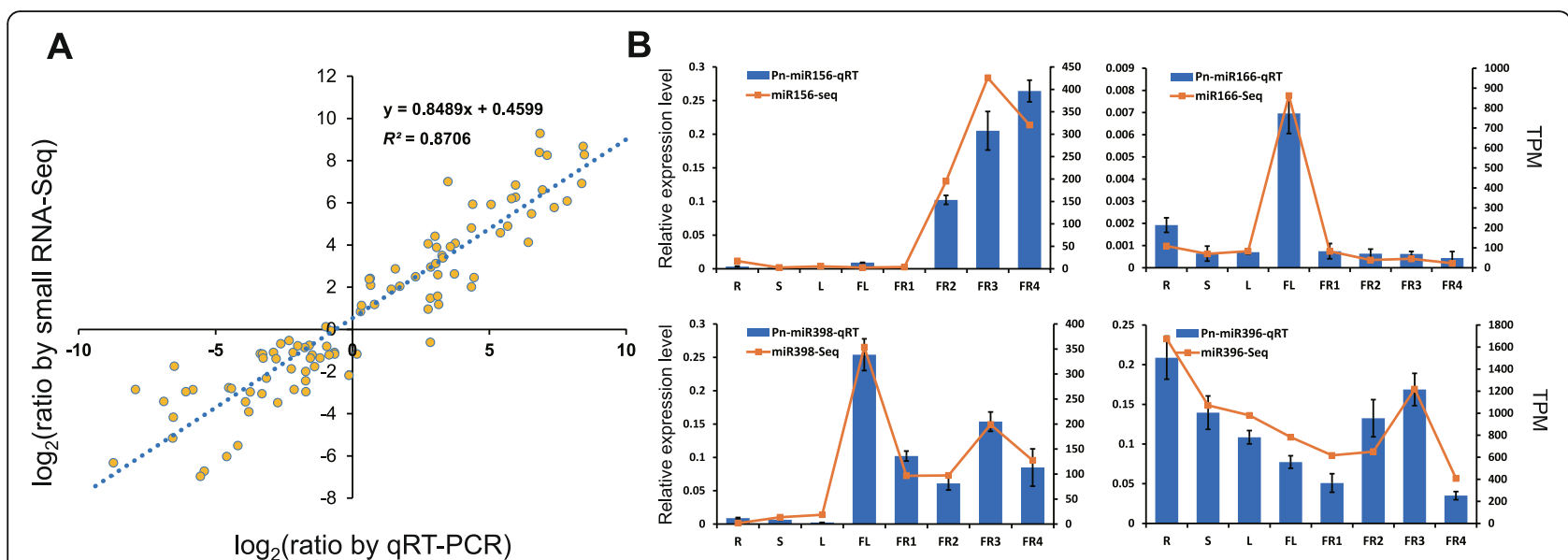

Fig. 4 qRT-PCR validation of the miRNA expression levels detected by small RNA sequencing. A. Correlations of miRNA expression profiles between small RNA sequencing and qRT-PCR. Log $_{2}$ (ratios) of qRT-PCR and small RNA-Seq were used to draw the scatter diagram. The trend line, regression equation and correlation coefficient were generated automatically in EXCEL. B. qRT-PCR analysis of miR156, miR166, miR398 and miR396 in P. nigrum. PnHis3 was used as an internal control. Data are the means \pm SD from 3 biological replicates

target CUAO and TYDC participate in alkaloid metabolism (tropane, piperidine and pyridine alkaloid biosynthesis and isoquinoline alkaloid biosynthesis). One of the 4 miRNAs, miR169, regulated the 4 homologous genes of UGT79B1 (Pn21.922, Pn21.931, Pn21.925 and $P n 21.928)$, which are involved in anthocyanin biosynthesis.
Expression analysis and 5' RLM-RACE validation of candidate miRNAs and their targets related to piperine biosynthesis

According to published studies, two precursors for piperine biosynthesis, piperoyl-CoA and piperidine were generated from phenylamine and lysine metabolism, respectively [24]. In this study, a total of 8 targets $(4 C L$ (Pn9.356) was regulated by miR166; 4 PERs (Pn24.105,

A miRNA targets prediction and annotation.

\begin{tabular}{|c|c|c|c|c|c|}
\hline \multirow{3}{*}{$\begin{array}{l}\text { miRNAs } \\
125\end{array}$} & \multirow{2}{*}{ Targets } & \multicolumn{2}{|c|}{$\begin{aligned} \text { Cleavage sites } \\
\end{aligned}$} & & \\
\hline & & Target Finde & r psRobot & Total & \\
\hline & 1,007 & 1,279 & 1,443 & 2,722 & \\
\hline Nr-Annote & KEG & Annotated & GO-Annotated & GO \& KEGG annotated & Overall \\
\hline 875 & 326 & & 322 & 377 & 884 \\
\hline $86.89 \%$ & 32.3 & & $31.97 \%$ & $37.43 \%$ & $87.79 \%$ \\
\hline
\end{tabular}

$B$

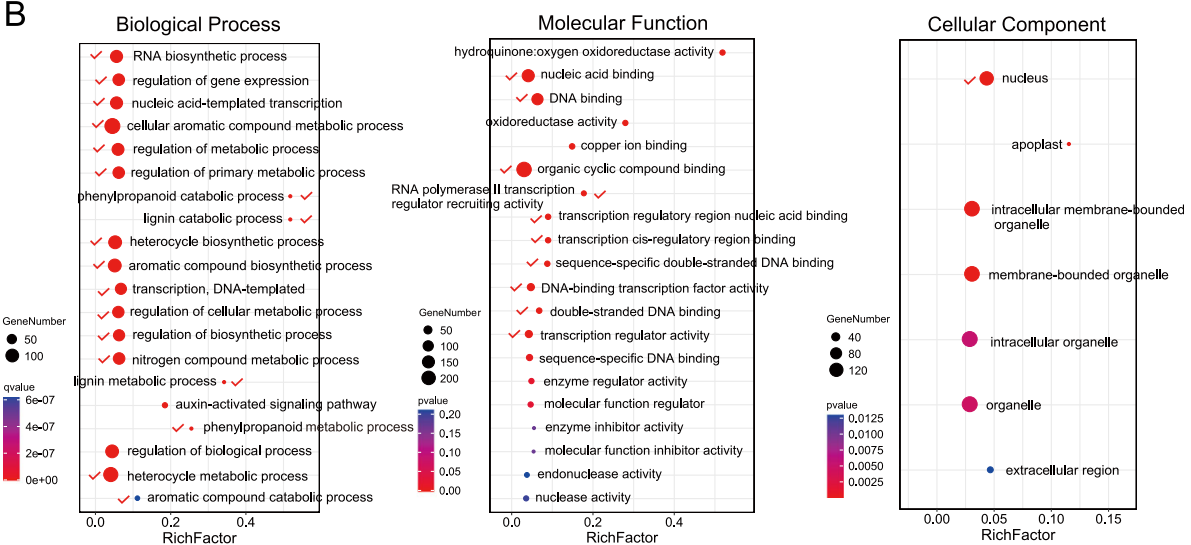

Fig. 5 Gene Ontology (GO) and KEGG annotation of predicted targets by TargetFinder and psRobot. A. MiRNA target prediction and annotation by TargetFinder and psRobot. B. GO enrichment of targets. Targets related to metabolic or transcriptomic processes are highlighted by red ticks. Ball size represents target numbers. The colours from red to blue or green represent $P$-values ranging from low to high 
Table 2 MiRNAs and corresponding targets involved in 5 secondary metabolic pathways of interest

\begin{tabular}{llll}
\hline Pathway & miRNAs & Annotations & Target ID \\
\hline Phenylpropanoid biosynthesis & miR166 & 4CL & Pn9.356 \\
Pn24.105; Pn7.365; & Pn16.989; Pn4.2519 \\
& miR396 & PER & Pn15.2487 \\
Tropane, piperidine and pyridine alkaloid biosynthesis & miR397 & CCR & Pn2.2577, Pn2.2494 \\
Isoquinoline alkaloid biosynthesis & miR159 & CUAO & Pn6.2746 \\
Anthocyanin biosynthesis & novel_mir15 & TYDC & CUAO Pn2.2577, Pn2.2494 \\
Phenylalanine, tyrosine and tryptophan biosynthesis & miR169 & UGT79B1/A3G2XYLT & Pn21.922; Pn21.931; \\
& & & Pn21.925; Pn21.928 \\
Phenylalanine metabolism & miR5021 & CM & Pn8.1140 \\
& novel_mir11 & AroB/DHQS & Pn16.732; Pn24.508 \\
& novel_mir18 & TRPE & Pn1.2305 \\
& miR166 & 4CL & Pn9.356 \\
\hline
\end{tabular}

Pn7.365, $P n 16.989$ and $P n 4.2519)$ were regulated by miR396; CCR (Pn15.2487) was regulated by miR397; CUAO (Pn2.2577, Pn2.2494) were regulated by novel_ mir15), which were found to directly participate in phenylamine and lysine metabolism, implying that these targets might be involved in the biosynthesis of piperine. To validate the expression relationships between these miRNAs and targets and to characterize the predicted cleavage sites, qRT-PCR and 5' RLM-RACE were performed. Finally, 3 targets $(4 C L, P E R, C C R)$ were found to have relatively opposite expression trends with their miRNAs (Fig. 6). The cleavage sites validated by $5^{\prime}$ RLM-RACE generally agreed with the predicted results (Fig. 6). In addition, we found that $4 C L$ and $C C R$ were both highly expressed in fruits compared with other tissues, and the corresponding miRNAs showed a relatively lower expression trend, which indicated that the metabolic processes from phenylalanine to caffeyl alcohol, coniferyl alcohol, 5-OH coniferyl alcohol and sinapyl alcohol were enhanced. On the other hand, the expression level of $P E R$ was low in the fruits, implying that the biosynthesis of lignin might be suppressed. Thus, these findings suggest that piperine biosynthesis was enhanced in the fruits under the regulation of miRNAs.

\section{Various miRNAs and targets participate in regulating piperine biosynthesis}

The simplified schematic in Fig. 7 shows the regulation of miRNAs and their targets participating in piperine biosynthesis in black pepper. Piperine is generated from piperoyl-CoA and $\Delta^{1}$-piperideine [24]. The enzyme catalysing piperine synthesis is known as 'piperoyl-CoA:piperidine $\mathrm{N}$-piperoyltransferase'. Piperoyl-CoA is derived from the phenylalanine metabolism pathway, but the direct precursors still unclear. In addition, lignin monomers also come from phenylalanine, and we suspect that the biosynthesis of piperine and lignin share the same origin to some degree. The fruits of black pepper have a highly lignified seed coat, which reminds us of the potential possible relationships under lignin and piperine
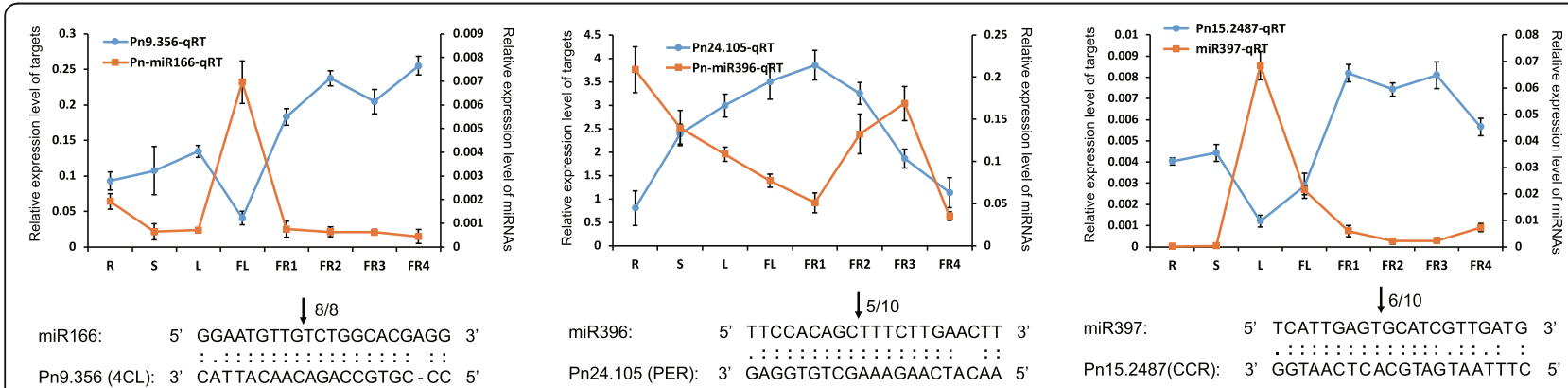

Fig. 6 qRT-PCR and 5' RLM-RACE validations of miRNAs and targets related to piperine biosynthesis. Arrows indicate the cleavage sites for miRNA with the number above suggesting the ratio of corrected cleavage events (out of 10) validated by $5^{\prime}$ RLM-RACE. PnHis 3 was used as an internal control 


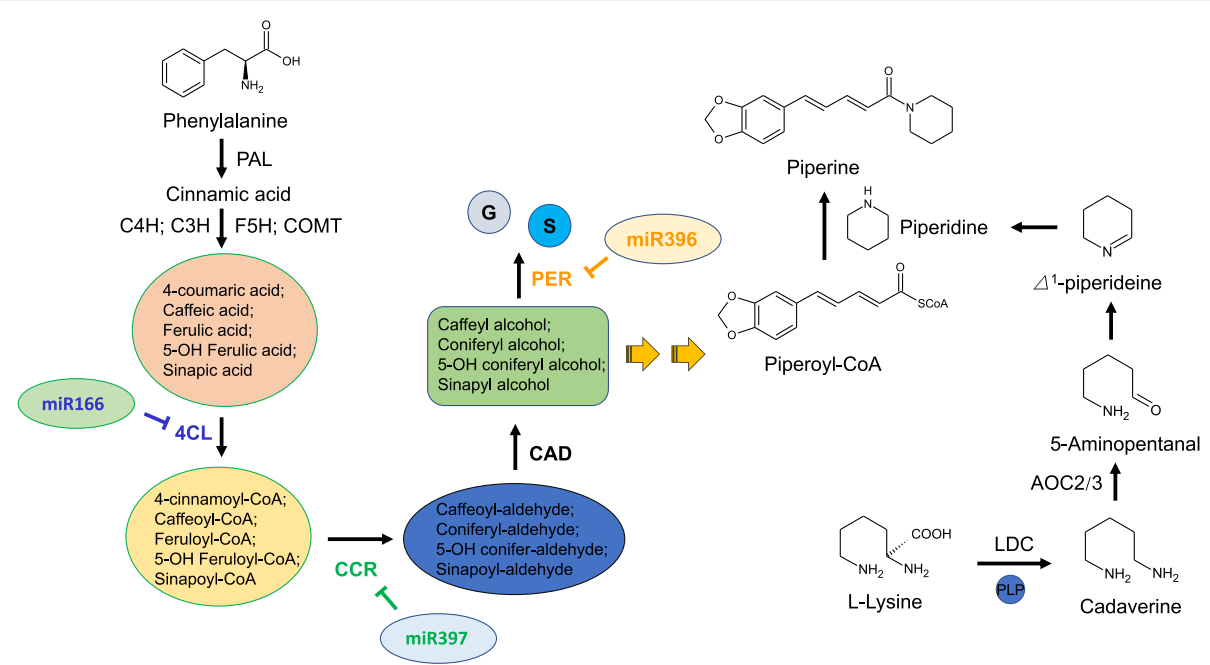

Fig. 7 Schematic showing miRNAs and targets involved in piperine biosynthesis. G: G lignin monomers; S: S lignin monomers. Chemical structures were drawn using Chemsketch 2.0

biosynthesis. In this study, we found that 3 miRNAs (miR166, miR397, and miR396) can regulate 4CL, CCR and $P E R$, thereby participating in the regulation of piperoyl-CoA biosynthesis. Furthermore, the enzyme that initiates the synthesis of piperidine originating from lysine and the enzymes that catalyse the last two steps from 5-aminopentanal to piperidine have not been identified.

\section{Discussion}

Piperine is the main pungent component present in the spice berries of black pepper, and it has been confirmed to have various bioactivities, such as immunomodulatory, anti-asthmatic, stimulatory, anticancer and antimicrobial properties [25-27]. MiRNAs regulating targets involved in secondary metabolism have been widely found in many plant species in recent years $[7,12,13$, 28], but few studies have investigated black pepper. In this study, we identified miRNAs related to plant development and secondary metabolism via small RNA sequencing in 8 tissues from black pepper, which provides new insights into miRNA functions in regulating secondary metabolites and alkaloid biosynthesis in plants.

\section{MiRNAs participating in alkaloids biosynthesis}

Alkaloids are low-molecular-weight nitrogenous organic compounds that are mostly derived from amino acids and are highly diverse and heterogeneous in nature. Alkaloids have a variety of biological activities and are used as pharmaceuticals, stimulants, narcotics, and poisons. MiRNAs regulating the biosynthesis of alkaloids have been reported in some species in recent years. In opium poppy, three miRNAs, pso-miR13, pso-miR2161 and pso-miR408, have been found to be involved in benzylisoquinoline alkaloid biosynthesis by regulating three functional enzymes, 7-Omethyltransferase (7-OMT), S-adenosyl-L-methionine:3'hydroxyN-methylcoclaurine 4'-O-methyltransferase 2 (4-OMT) and reticuline oxidase-like protein (in charge of the conversion of S-reticuline to (S)-scoulerine in benzylisoquinoline alkaloid biosynthesis), respectively [12]. In tobacco, 4 unique tobacco-specific miRNAs have been predicted to target key genes of the nicotine biosynthesis and catabolism pathways; nta-miRX17 targets quinolinate phosphoribosyltransferase 1 (QPT1); nta-miRX27 targets QPT2; nta-miRX20 targets CYP82E4; and nta-miRX19 targets NAC_148 [13]. Moreover, the expression of nta-miRX27 was found to be inhibited by an endogenous target mimic (eTM), nta-eTMX27, resulting in the upregulation of $Q P T 2$, thereby enhancing nicotine biosynthesis in toppingtreated tobacco plants. In our study, 3 miRNAs and their targets were found to be involved in the biosynthesis of piperine (Fig. 7); miR166 targets $4 C L$; miR396 targets PER; and miR397 targets CCR. In previous studies, $4 C L$ was predicted to be regulated by miR156, miR1858 and miR396 [29, 30], although without any experimental evidence. On the other hand, the biosynthesis of piperine was derived from phenylalanine, which is also the precursor for many secondary metabolites, such as lignans, anthocyanins, and flavonoids. In our findings, the upregulated miR396 in fruits resulted in a relative downregulation of $P E R$, which mediated the biosynthesis of lignin monomers (Fig. 6). At the same time, the expression of $4 C L$ and $C C R$ was enhanced, while their corresponding miRNAs showed downregulation in fruits (Fig. 6). Our findings indicate that numerous miRNAs, as well as their functional enzyme targets, participate in balancing the biosynthesis 
of lignin and piperine in the fruits of black pepper. In addition, several other studies on different species have reported the miRNAs involved in alkaloid biosynthesis $[8,31,32]$. In conclusion, miRNAs always function as regulators in balancing plant growth, stress resistance and metabolism processes including the synthesis of various alkaloids in different species.

\section{MiRNAs modulate secondary metabolism in plants}

In previous studies, many miRNAs have been found to function directly or indirectly in regulating secondary metabolism in plants. In Arabidopsis, miR156 has been found to target SPLs, which indirectly regulates the accumulation of anthocyanins by preventing the expression of anthocyanin biosynthetic genes through destabilization of a MYB-bHLH-WD40 transcriptional activation complex [6]. Interestingly, miR156-targeted SPL can also progressively regulate sesquiterpene biosynthesis in Arabidopsis by directly binding the promoter of TPS21 (sesquiterpene synthase gene) and activating its expression [11], which indicates that the same miRNA and its target can take part in regulating multiple metabolic processes. In addition, miR828 and miR858 have also been found to be involved in regulating anthocyanin and flavonol accumulation by target VvMYB114 [7]. In our study, miR169 participated in anthocyanin biosynthesis in black pepper by target UGT79B1 (Table 2), an anthocyanidin 3-O-glucoside 2 "'-O-xylosyltransferase catalysing pelargonidin 3-Oglucoside to pelargonidin 3-O-beta-D-sambubioside. In our study, 74 miRNAs were identified as targeting 111 genes involved in metabolic pathways (Supplementary Table 7), indicating the powerful functions of miRNAs in regulating the metabolic pathway in black pepper. With the development of high-throughput sequencing technology, an increasing number of miRNAs have been found to participate in regulating various primary or secondary metabolic processes in plants. For example, the overexpression of miR8154 and miR5298b in Taxus cells resulted in the upregulation of key genes in Taxol, phenylpropane, and flavonoid biosynthesis [9]. In persimmon, miR395p-3p can target bHLH mRNA, and in turn, regulate the structural genes that influence proanthocyanidin accumulation [10]. In $H$. capsica, miR6194 can target $\mathrm{F} 3 \mathrm{H}$, which is a key enzyme in the biosynthesis of flavonols, anthocyanidins and proanthocyanidins [33]. In addition, many miRNAs have been identified that are involved in lignin synthesis, sugar and acid metabolism, and hormone signalling during pear fruit development [29]. These findings suggested that miRNA is an important regulator for controlling metabolic processes at the posttranscriptional level.

\section{Conclusions}

As a common phenomenon in plants, miRNA regulation of secondary metabolism enhances the ability of plants to respond to the environment. Our work has proven that various miRNAs involved in the biosynthesis of many secondary metabolites in black pepper, especially in regulating piperine, a special alkaloid present in plants within the Piper genus, which provides different perspectives and abundant information on the regulation of plant metabolism and development by miRNAs.

\section{Methods}

\section{Plant materials and total RNA extraction}

Eight tissues (root, stem, leaf, flower, and the fruits from $2,4,6$, and 8 months after pollination) were collected from 13 year-old black pepper cultivars (Piper nigrum L. cv. 'Reyin No.1') from the Spice and Beverage Research Institute of Chinese Academy of Tropical Agricultural Science, Wanning, Hainan, China. All samples were harvested in the field at 10:00 a.m., frozen in liquid nitrogen immediately and then sent to Novogene (Beijing, China) for RNA extraction and library construction. A total of 24 samples with 3 biological replicates were prepared.

Total RNA was prepared using the miRcute Plant miRNA Isolation Kit DP504 (Tiangen, Beijing, China) according to the manufacturer's instructions. The extracted total RNA was then monitored by an Agilent Bioanalyzer 2100 system (Agilent Technologies, CA, USA), $1 \%$ agarose gels and a NanoPhotometer ${ }^{\circ}$ spectrophotometer (IMPLEN, CA, USA) for integrity, purity and concentration examination to guarantee highquality total RNA for small RNA sequencing.

\section{Small RNA library construction}

Approximately $3 \mu \mathrm{g}$ of total RNA from each sample was prepared for small RNA library construction. In brief, the total RNA was first purified by polyacrylamide gel electrophoresis (PAGE) to obtain small RNAs that were 18-30 nt in length. Then, the NEB 3' SR adaptor was ligated to the 3 ' end of the small RNA fragments; doublestranded DNA adaptor was transformed; 5' end adapters were then ligated to the $5^{\prime}$ ends of the small RNA fragments; and cDNA was generated by a reverse transcription reaction. Several rounds of PCR amplification were performed, and the products were purified by agarose gel to obtain enough fragments from 100 to $120 \mathrm{bp}$ for Illumina sequencing. The quality of purified products was assessed using the Agilent Bioanalyzer 2100 system.

\section{Bioinformatics analysis}

The raw reads of small RNA sequencing were first filtered to obtain clean data for later analysis via the following steps: removing low quality reads, reads containing poly$\mathrm{N}$, reads shorter than $18 \mathrm{nt}$, reads with $5^{\prime}$ adapter 
contaminants and reads without a $3^{\prime}$ adapter insert. Then, all clean reads were aligned to the $P$. nigrum genome [34] by Bowtie2 [35]. Mapped small RNA tags were first used for secondary structure prediction and then aligned to the miRBase21.0 [36] database (http://www.mirbase.org/) for known miRNA identification. At the same time, all clean reads were aligned to the GenBank [37] (ftp://ftp.ncbi. nlm.nih.gov/genbank/) and Rfam 12.1 [38] (http://rfam. janelia.org/) databases to annotate other small RNAs, including rRNA, tRNA, small nuclear RNA (snRNA) and small nucleolar RNA (snoRNA). Reads mapped to the genome without any annotations were then used for novel miRNA prediction by mirDeep2 [39] and PIPmiR1.1 [40]. Subsequently, psRobot [41] and TargetFinder [42] were used for computational prediction of the miRNA targets. The expression level for all identified miRNAs was calculated by transcripts per million (TPM) using the following formula: mapped read count/total reads $\times 1,000,000$. To evaluate the expression changes between samples, 3 biological replicates were first merged by the mean values of TPM (mTPM) and then calculated by $\log _{2}$ (mTPM of sample $1 /$ mTPM of sample 2 ). A heatmap was generated by Genesis software (http://genome.tugraz.at/) using the fold change values.

\section{Gene ontology and KEGG pathway enrichment}

To explore the functional category distribution and pathway enrichment of the miRNA targets, Gene Ontology (GO) [43] term analysis (www.geneontology.org) and KEGG [22] analysis were performed using Blast2GO [21] software and KOBAS 3.0 [44] (http://kobas.cbi.pku. edu.cn/). All GO categories and KEGG pathways were screened under the condition of a $P$-value $<0.05$.

\section{5' RLM-RACE used for target cleavage site identification}

To verify the predicted target cleavage sites, RNA ligasemediated rapid amplification of the cDNA ends (RLMRACE) was performed using the FirstChoice ${ }^{\bullet}$ RLM-RACE Kit AM1700 (Invitrogen, Thermo Fisher Scientific, Massachusetts, USA). Briefly, the protocol from the manufacturer's instructions involved following steps: (1) total RNA (5 $\mu \mathrm{g})$ from all samples (24 samples containing biological repeats) were equally mixed together; (2) RNA mixtures were treated with tobacco acid pyrophosphatase (TAP) to remove the $5^{\prime}$ cap from the mRNA; (3) a $5^{\prime}$ RACE adapter was ligated to decapped mRNA without calf intestinal phosphatase (CIP) treatment; (4) reverse transcription was performed by GeneRacer Oligo dT primers to produce cDNA; (5) PCR amplification was performed with adapter primers and 3' gene-specific primers (designed from 3' UTR); and (6) PCR products were then ligated to pGEM T-easy vector (Promega, Madison, WI, USA) for later sequencing by TsingKe (Beijing, China).
Primers were designed by Primer 5.0 and synthesized by Tianyi Huiyuan, Wuhan, China.

\section{Quantitative real-time reverse transcriptase-polymerase chain reaction (qRT-PCR)}

To quantify the expression levels of miRNAs and target genes, stem-loop RT-PCR was performed according to reported methods $[45,46]$. The reverse transcription reaction was performed as follows: (1) $2 \mu \mathrm{g}$ of total RNA was first mixed with the primers $(1 \mu$ l Oligo dT primers and $1 \mu \mathrm{l}$ stem-loop primers), $1 \mu \mathrm{l}$ of $\mathrm{dNTP}$ and appropriate RNA-free water up to $13 \mu \mathrm{l}$; (2) the mixture was incubated at $65^{\circ} \mathrm{C}$ for $5 \mathrm{~min}$ to align primers to the RNA, and it was transferred to ice immediately for $2 \mathrm{~min}$; (3) $4 \mu \mathrm{l}$ of $5 \times$ FS buffer, $1 \mu \mathrm{l}$ RNaseOUT (40 units/ $\mu \mathrm{l}$ ), $1 \mu \mathrm{l}$ of DTT and $1 \mu \mathrm{l}$ of SuperScript III RT (200 units/ $\mu$ l) were added to the mixture in a $20-\mu$ l reaction solution; (4) reverse transcription reactions were performed via the following steps: $16^{\circ} \mathrm{C}$ for $30 \mathrm{~min}, 60$ cycles of $30^{\circ} \mathrm{C}$ for $30 \mathrm{~s}$, $42^{\circ} \mathrm{C}$ for $30 \mathrm{~s}, 50^{\circ} \mathrm{C}$ for $1 \mathrm{~s}$, and then $85^{\circ} \mathrm{C}$ for $5 \mathrm{~min}$ to stop the reactions. For the qRT-PCR experiments, cDNA products were first diluted into $50 \times$ solutions. Subsequently, $10 \mu \mathrm{l}$ of diluted cDNA was combined with $10 \mu \mathrm{l}$ of FastStart Essential DNA Green Master (Roche, Basel, Switzerland) qRT-PCR master mix containing $0.25 \mu \mathrm{M}$ forward and reverse primers. qRT-PCR was performed in a QuantStudio 5 system (Thermo Fisher Scientific, Massachusetts, USA) with 40 cycles of $95^{\circ} \mathrm{C}$ for $5 \mathrm{~s}$ and $60{ }^{\circ} \mathrm{C}$ for $30 \mathrm{~s}$. PnHis3 was used as the internal control. The primers used in this study are listed in Supplementary Table 9.

\section{Abbreviations}

4CL: 4-coumarate--CoA ligase; 5'-RLM-RACE: 5' RNA ligase-mediated rapid amplification of CDNA ends; AMIE: Amidase; AroB/DHQS: 3-dehydroquinate synthase; CCR: Cinnamoyl-CoA reductase; CIP: Calf intestinal phosphatase;

CM: Chorismate mutase; CUAO: Copper amine oxidase; DFR: Dihydroflavonol reductase; $\mathrm{DHQ}-\mathrm{SDH}$ : 3-dehydroquinate dehydratase / shikimate dehydrogenase; F3'H: Flavonoid 3'-hydroxylase; GO: Gene Ontology; KEGG: Kyoto Encyclopedia of Genes and Genomes; LDC: Lysine decarboxylase; miRNA: microRNA; NADPH: Nicotinamide adenine dinucleotide phosphate; PAL: Phenylalanine ammonia lyase; PER: Peroxidase; PLP: Pyridoxal phosphate; qRT-PCR: Quantitative real-time reverse transcriptase-polymerase chain reaction; snRNA: Small nuclear RNA; snoRNA: Small nucleolar RNA; TAP: Tobacco acid pyrophosphatase; TPM: Transcripts Per Kilobase Million; TPS21: Terpene synthases 21; TRPE: Anthranilate synthase component I; TYDC: Tyrosine decarboxylase; UGT79B1/A3G2XYLT: Anthocyanidin 3-O-glucoside 2"'-O-xylosyltransferase

\section{Supplementary Information}

The online version contains supplementary material available at https://doi. org/10.1186/s12864-021-08154-4.

\section{Additional file 1.}

Additional file 2.

Additional file 3.

Additional file 4.

Additional file 5 . 


\section{Additional file 6 . \\ Additional file 7. \\ Additional file 8. \\ Additional file 9. \\ Additional file 10 \\ Additional file 11. \\ Additional file 12 .}

\section{Acknowledgements}

Not applicable.

\section{Authors' contributions}

Chaoyun Hao and Jie Luo conceived the study. Yuanhao Ding, Haiyan Hu and Jie Luo designed the experiments. Yuanhao Ding, Yuyuan Mao and Yi Cen performed the experiments. Yuanhao Ding and Haiyan Hu wrote the manuscript. Yuefeng Su, Xuemin Ma and Lisong Hu provide suggestions for the experiments. Xuemin Ma, Haiyan Hu, Lu Long and Jie Luo revised the manuscript. The author(s) read and approved the final manuscript.

\section{Funding}

This research was supported by the Major Science and Technology Programme of Ningxia Hui Autonomous Region (2016BZ06), the Hainan Provincial Postdoctoral Science Foundation of China (BSH-RST-2019002) and the Central Public-interest Scientific Institution Basal Research Fund for Chinese Academy of Tropical Agricultural Sciences (No. 1630142019001).

\section{Availability of data and materials}

The small RNA sequencing data used in this study can be found in the National Center for Biotechnology Information (NCBI) SRA database under accession number: PRJNA589468.

\section{Declarations}

\section{Ethics approval and consent to participate}

Use of all plant materials in this study complied with relevant institutional, national, and international guidelines and legislation. Tissues of black pepper cultivars (Piper nigrum L. cv. 'Reyin No.1') were permitted to collected from the Spice and Beverage Research Institute of Chinese Academy of Tropical Agricultural Science, Wanning, Hainan, China.

\section{Consent for publication}

Not applicable.

\section{Competing interests}

The authors declare that they have no competing interests.

\section{Author details \\ 'Hainan Key Laboratory for Sustainable Utilization of Tropical Bioresource, School of Tropical Crops, Hainan University, Haikou 570228, China. ${ }^{2}$ Spice and Beverage Research Institute, Chinese Academy of Tropical Agricultural Sciences, Wanning 571533, Hainan, China. ${ }^{3}$ Ministry of Agriculture Key Laboratory of Genetic Resources Utilization of Spice and Beverage Crops, Wanning 571533, Hainan, China. ${ }^{4}$ State Key Laboratory of Cotton Biology, Key Laboratory of Plant Stress Biology, School of Life Science, Henan University, Kaifeng 475004, Henan, China. ${ }^{5}$ Hainan Provincial Key Laboratory of Genetic Improvement and Quality Regulation for Tropical Spice and Beverage Crops, Wanning 571533, Hainan, China.}

\section{Received: 20 March 2021 Accepted: 3 November 2021}

\section{Published online: 19 November 2021}

\section{References}

1. Murlidhar MK. GT: Piper nigrum and Piperine: An Update. Phytother Res. 2013;27(8):1121-30.

2. Kato Massuo J, Furlan M. Chemistry and evolution of the Piperaceae. Pure Appl Chem. 2007;79:529.
3. De A. Morandim A, DCB B, Kato MJ, Cavalheiro AJ, Da S. Bolzani V, Furlan M. Circadian rhythm of anti-fungal prenylated chromene in leaves of Piper aduncum. Phytochem Anal. 2005;16(4):282-6.

4. Dewick PM. Medicinal Natural Products: A Biosynthetic Approach. 3rd ed. United Kingdom: John Wiley \& Sons Ltd; 2009. p. 21, 147-53. 166$169,326-328$

5. Lv Z, Wang Y, Liu Y, Peng B, Zhang L, Tang K, et al. The SPB-box transcription factor AaSPL2 positively regulates artemisinin biosynthesis in Artemisia annua L. Front Plant Sci. 2019;10:409.

6. Jin-Ying G, Felippes FF, Chang-Jun L, Detlef W, Jia-Wei W. Negative regulation of anthocyanin biosynthesis in Arabidopsis by a miR156-targeted SPL transcription factor. Plant Cell. 2011;23(4):1512-22.

7. Tirumalai V, Swetha C, Nair A, Pandit A, Shivaprasad PV. miR828 and miR858 regulate VVMYB114 to promote anthocyanin and flavonol accumulation in grapes. J Exp Bot. 2019.

8. Biswas S, Hazra S, Chattopadhyay S. Identification of conserved miRNAs and their putative target genes in Podophyllum hexandrum (Himalayan Mayapple). Plant Gene. 2016;6(C):82-9.

9. Zhang M, Dong Y, Nie L, Lu M, Fu C, Yu L. High-throughput sequencing reveals miRNA effects on the primary and secondary production properties in long-term subcultured Taxus cells. Front Plant Sci. 2015;6:604.

10. Luo Y, Zhang X, Luo Z, Zhang Q, Liu J. Identification and characterization of microRNAs from Chinese pollination constant non-astringent persimmon using high-throughput sequencing. BMC Plant Biol. 2015;15:11.

11. Yu Z-X, Wang L-J, Zhao B, Shan C-M, Zhang Y-H, Chen D-F, et al. Progressive regulation of Sesquiterpene biosynthesis in Arabidopsis and patchouli (Pogostemon cablin) by the miR156-targeted SPL transcription factors. Mol Plant. 2015;8(1):98-110.

12. Boke H, Ozhuner E, Turktas M, Parmaksiz I, Ozcan S, Unver T. Regulation of the alkaloid biosynthesis by miRNA in opium poppy. Plant Biotechnol $\mathrm{J}$. 2015;13(3):409-20.

13. Li F, Wang W, Zhao N, Xiao B, Cao P, Wu X, et al. Regulation of nicotine biosynthesis by an endogenous target mimicry of MicroRNA in tobacco. Plant Physiol. 2015;169(2):1062-71.

14. Asha S, Soniya EV. Transfer RNA derived small RNAs targeting defense responsive genes are induced during Phytophthora capsici infection in black pepper (Piper nigrum L.). Front Plant Sci. 2016;7(767).

15. Asha S, Nisha J, Soniya EV. In silico characterisation and phylogenetic analysis of two evolutionarily conserved miRNAs (miR166 and miR171) from black pepper (Piper nigrum L.). Plant Mol Biol Report. 2013;31(3):707-18.

16. Asha S, Sreekumar S, Soniya EV. Unravelling the complexity of microRNAmediated gene regulation in black pepper (Piper nigrum L.) using highthroughput small RNA profiling. Plant Cell Rep. 2016;35(1):53-63.

17. Joy N, Soniya EV. Identification of an miRNA candidate reflects the possible significance of transcribed microsatellites in the hairpin precursors of black pepper. Funct Integr Genomics. 2012;12(2):387-95.

18. Ding Y, Ma Y, Liu N, Xu J, Hu Q, Li Y, et al. microRNAs involved in auxin signalling modulate male sterility under high-temperature stress in cotton (Gossypium hirsutum). Plant J. 2017;91(6):977-94.

19. Liu N, Tu L, Tang W, Gao W, Lindsey K, Zhang X. Small RNA and degradome profiling reveals a role for miRNAs and their targets in the developing fibers of Gossypium barbadense. Plant J. 2014;80(2):331-44.

20. Kratz A, Tomita M, Krishnan A. GeNESiS: gene network evolution simulation software. BMC Bioinformatics. 2009;9:541.

21. Conesa A, Götz S, García-Gómez JM, Terol J, Talón M, Robles M. Blast2GO: a universal tool for annotation, visualization and analysis in functional genomics research. Bioinformatics (Oxford, England). 2005;21(18):3674-6.

22. Kanehisa M, Goto S. KEGG: Kyoto encyclopedia of genes and genomes. Nucleic Acids Res. 2000;28(1):27-30.

23. Guo A-Y, Chen X, Gao G, Zhang H, Zhu Q-H, Liu X-C, et al. PlantTFDB: a comprehensive plant transcription factor database. Nucleic Acids Res. 2007; 36(suppl_1):D966-9.

24. Okwute SK, Egharevba HO. Piperine-type amides: review of the chemical and biological characteristics. Int J Chem. 2013;5(3).

25. Umadevi P, Soumya M, George JK, Anandaraj M. Proteomics assisted profiling of antimicrobial peptide signatures from black pepper (Piper nigrum L.). Physiol Mol Biol Plants. 2018;24(3):379-87.

26. Manayi A, Nabavi SM, Setzer WN, Jafari S. Piperine as a potential anticancer agent: a review on preclinical studies. Curr Med Chem. 2018; 25(37):4918-28. 
27. Xie Z, Wei Y, Xu J, Lei J, Yu J. Alkaloids from Piper nigrum synergistically enhanced the effect of paclitaxel against paclitaxel-resistant cervical Cancer cells through the downregulation of Mcl-1. J Agric Food Chem. 2019;67(18): 5159-68.

28. Zhao S, Wang X, Yan X, Guo L, Mi X, Xu Q, et al. Revealing of MicroRNA involved regulatory gene networks on Terpenoid biosynthesis in Camellia sinensis in different growing time points. J Agric Food Chem. 2018;66(47): 12604-16.

29. Wu J, Wang D, Liu Y, Wang L, Qiao X, Zhang S. Identification of miRNAs involved in pear fruit development and quality. BMC Genomics. 2014;15:953.

30. Singh N, Sharma A. Turmeric (Curcuma longa): miRNAs and their regulating targets are involved in development and secondary metabolite pathways. Comptes Rendus Biologies. 2017;340(11-12):481-91.

31. Pani A, Mahapatra RK. Computational identification of microRNAs and their targets in Catharanthus roseus expressed sequence tags. Genomics Data. 2013;1:2-6.

32. Prakash $P$, Rajakani $R$, Gupta V. Transcriptome-wide identification of Rauvolfia serpentina microRNAs and prediction of their potential targets. Comput Biol Chem. 2016;61:62-74.

33. Yang $R$, Zeng $Y$, Yi X, Zhao L, Zhang $Y$. Small RNA deep sequencing reveals the important role of microRNAs in the halophyte Halostachys caspica. Plant Biotechnol J. 2015:13(3):395-408.

34. Hu L, Xu Z, Wang M, Fan R, Yuan D, Wu B, et al. The chromosome-scale reference genome of black pepper provides insight into piperine biosynthesis. Nat Commun. 2019;10(1):4702.

35. Langmead B, Trapnell C, Pop M, Salzberg SL. Ultrafast and memory-efficient alignment of short DNA sequences to the human genome. Genome Biol. 2009;10(3):R25.

36. Griffiths-Jones S. miRBase: the microRNA sequence database. Methods Mol Biol (Clifton, NJ). 2006;342:129-38.

37. Sayers EW, Cavanaugh M, Clark K, Ostell J, Pruitt KD, Karsch-Mizrachi I. GenBank. Nucleic Acids Res. 2019;47(D1):D94-d99.

38. Nawrocki EP, Burge SW, Bateman A, Daub J, Eberhardt RY, Eddy SR, et al. Rfam 12.0: updates to the RNA families database. Nucleic Acids Res. 2014; 43(D1):D130-7.

39. An J, Lai J, Lehman ML, Nelson CC. miRDeep*: an integrated application tool for miRNA identification from RNA sequencing data. Nucleic Acids Res. 2012;41(2):727-37.

40. Breakfield NW, Corcoran DL, Petricka JJ, Shen J, Sae-Seaw J, Rubio-Somoza I, et al. High-resolution experimental and computational profiling of tissuespecific known and novel miRNAs in Arabidopsis. Genome Res. 2012;22(1): 163-76.

41. Wu HJ, Ma YK, Chen T, Wang M, Wang XJ. PsRobot: a web-based plant small RNA meta-analysis toolbox. Nucleic Acids Res. 2012;40(Web Server issue):W22-8.

42. Bo X, Wang S. TargetFinder: a software for antisense oligonucleotide target site selection based on MAST and secondary structures of target mRNA. Bioinformatics (Oxford, England). 2005;21(8):1401-2.

43. Ashburner M, Ball CA, Blake JA, Botstein D, Butler H, Cherry JM, et al. Gene ontology: tool for the unification of biology. The Gene Ontology Consortium. Nat Genet. 2000;25(1):25-9.

44. Bu D, Luo H, Huo P, Wang Z, Zhang S, He Z, et al. KOBAS-i: intelligent prioritization and exploratory visualization of biological functions for gene enrichment analysis. Nucleic Acids Res. 2021;49(W1):W317-w325.

45. Varkonyi-Gasic E, Wu R, Wood M, Walton EF, Hellens RP. Protocol: a highly sensitive RT-PCR method for detection and quantification of microRNAs. Plant Methods. 2007:3:12

46. Jin C, Sun Y, Shi Y, Zhang Y, Chen K, Li Y, et al. Branched-chain amino acids regulate plant growth by affecting the homeostasis of mineral elements in rice. Sci China Life Sci. 2019;62(8):1107-10.

\section{Publisher's Note}

Springer Nature remains neutral with regard to jurisdictional claims in published maps and institutional affiliations.

Ready to submit your research? Choose BMC and benefit from:

- fast, convenient online submission

- thorough peer review by experienced researchers in your field

- rapid publication on acceptance

- support for research data, including large and complex data types

- gold Open Access which fosters wider collaboration and increased citations

- maximum visibility for your research: over $100 \mathrm{M}$ website views per year

At BMC, research is always in progress.

Learn more biomedcentral.com/submissions 